KARL EIBL

\title{
Epische Triaden. Über eine stammesgeschichtlich verwurzelte Gestalt des Erzählens
}

Eines der zentralen Probleme einer evolutionsbiologischen Deutung kultureller Phänomene ist die Unterscheidung von biologischer Grundausstattung (`Universalien`) und kultureller Modifikation. Denn bei den kulturell relevanten Eigenschaften und Verhaltensweisen ist die Grundausstattung bereits darauf angelegt, kulturell fertig gebaut zu werden, so dass sie nur in ihren kulturellen Manifestationen direkt sichtbar wird. Musterbeispiel ist die ,Universalgrammatikı, deren Existenz zwar mit guten Gründen postuliert werden kann, deren Beschreibung aber die größten Schwierigkeiten bereitet, weil sie nur als Grammatik von Einzelsprachen in die Erscheinung tritt. In eine ähnliche Problemzone führt der Gebrauch des psychologischen Gestalt-Begriffs, wenn man die evolutionären Grundlagen der Gestaltwahrnehmung ins Visier nimmt. Er ist zwar in der Tradition immer wieder einmal mit evolutionsbiologischen Überlegungen in Kontakt gebracht worden, hat aber ein so hohes Potential eigener sinnlicher Plausibilität, dass es hier eher bei gelegentlichen Aperçus geblieben ist. - Das ist der Rahmen, in dem hier die Frage gestellt wird: In welchem Sinne ist der Gestalt-Begriff für das Beschreibungsinventar eines evolutionären Ansatzes der Literaturwissenschaft brauchbar?

1.

Der Begriff der Gestalt hat seine Domäne vor allem im Bereich der visuellen Wahrnehmung. ${ }^{1}$ Das gilt sowohl für die Wahrnehmungspsychologie allgemein als auch für die Versuche, mit seiner Hilfe biologische Grundlagen künstlerischer Aktivitäten zu ermitteln. Hervorzuheben sind hier insbesondere die Arbeiten von Irenäus Eibl-Eibesfeldt. ${ }^{2}$ In der Literaturwissenschaft gehört der Begriff eher in eine (inzwi-

${ }^{1}$ Das Standardwerk E. Bruce Goldstein 2002 behandelt in sieben Kapiteln die visuelle Wahrnehmung, in dreien die auditive, in je einem die Lageorientierung, die haptische Wahrnehmung und die Geruchs- und Geschmackswahrnehmung.

${ }^{2}$ Ein Beitrag der Psychologie zur Kunsttheorie, der biologische Aspekte berücksichtigt, ist Schuster 2000 (Kunst ist hier selbstverständlich bildende Kunst). Monumental jetzt Eibl-Eibesfeldt/Sütterlin 2007, zur Gestaltwahrnehmung insbesondere 162-177. 
schen versandete) selbständige Tradition, in der die sorganischer Dualität von Gehalt und Gestalt die > positivistischer von Inhalt und Form ersetzen sollte. So ist denn für Oskar Walzel Gestalt »alles, was auf den äußeren oder den inneren Sinn wirkt, was zum Ohr oder Auge spricht oder auch Gehör- oder Gesichtsvorstellungen wachruft. (Walzel 1929, 178). Dabei werden dann ausdrücklich die "Abwege empirischer Seelenkunde« verurteilt (ibid., 12) - nun gut. Ich setze an bei Überlegungen von Eibl-Eibesfeldt, der den Gestaltbegriff in den Zusammenhang angeborener (phylogenetisch erworbener),Vorurteiler stellt. Mittels dieser angeborenen , Vorurteiles strukturieren wir unsere Erfahrungs- und Handlungsfelder. Es ist offenkundig, dass ein solches Vor-Urteil nicht auf sinnliche Qualitäten allein beschränkt werden kann, sondern auch deren kognitive Verknüpfung betrifft.

Setzt man dies voraus, dann gibt es eine Vielzahl angeborener Prinzipien, Interessen, Schemata - eben `Gestalten`, die beim Erzählen bzw. beim Vernehmen von Erzählungen aktiviert werden und in gewisser Weise das Erzählte überhaupt erst hervorbringen. Ihre Entdeckung ist dem Wirken von Philosophen zu danken, die sich zweieinhalb tausend Jahre um eine Aufdeckung und Erklärung der Prinzipien bemüht haben, denen das Denken aller Menschen folgt, längst ehe der moderne biologische Begriff vom Menschen diesen Bemühungen ein festeres und realwissenschaftlich verankertes Fundament geben konnte. Ein Beispiel ist das Kausalprinzip. Die kausale Verknüpfung von Sachverhalten ist eine evolutionär hoch bewährte Methode, um Wissensbestände in Regeln zu fassen und parat zu halten. ${ }^{3}$ Wenn wir hören: „Es war dunkel und er stolperte über einen Stein`, dann wissen wir, dass er stolperte, weil er nichts sah, und dass er nichts sah, weil es dunkel war. Die Information wird also von uns selbst mit Hilfe des Kausalprinzips generiert. Ein Prinzip von ähnlicher Allgemeinheit ist die Teleologie. Auch sie hat evolutionäre Wurzeln, denn es war schon immer ein Vorteil im Überlebens- und Reproduktionswettbewerb, wenn man die Dinge schnell hinsichtlich ihres möglichen Nutzens einschätzen konnte. Von größter Bedeutung für den Informationshaushalt ist das Prinzip der Widerspruchsfreiheit; denn Informationen, die widersprüchlich sind, sind als Handlungsanleitungen unbrauchbar (und das entscheidende, letztinstanzliche Selektionskriterium der Evolution ist das erfolgreiche Handeln, nicht die niveauvolle Reflexion). Schließlich nenne ich einleitend noch das Prinzip der Detektion: Das Aufdecken verborgener Ursachen als das Grundprinzip allen Erklärens ${ }^{4}$ dürfte gleichfalls zu unserer kognitiven Grundausstattung gehö-

${ }^{3}$ Wahrscheinlich ist diese Methode schon im vormenschlichen Bereich weit verbreitet, wenngleich die Individuen, die sie anwenden, uns darüber keine Auskunft geben. Wir rubrizieren das Verhalten von Pawlows Hund unter 'Konditionierung`. Aber wir könnten ihm ebenso gut eine kausale Verknüpfung zwischen dem Klingelton und dem Futter zuschreiben und das als Beleg dafür verwenden, dass auch Hunde kausal denken.

${ }^{4}$ Es ist kein Zufall, dass Karl R. Popper bei seiner klassisch gewordenen Explikation des Begriffs ,Erklären als Beispiel die Aufdeckung eines Giftmordes wählt: „Wir finden eine Leiche und wir wollen erklären, was denn hier geschehen ist.« (Popper 1972, 49). 
ren. Wir verwenden diese Organisationsmethoden nicht nur zur Verwaltung unseres Alltagswissens, sondern auch bei der Konstruktion erfundener Geschichten, indem wir den Stoff kausal verknüpfen, die Geschehnisse auf eine Pointe zulaufen lassen, Mehrdeutiges nach dem Prinzip der Widerspruchsfreiheit monosemieren und/oder Geheimnisse mittels analytischer Techniken enträtseln.

Gleich vorweg ist jedoch zur Vermeidung von Missverständnisse zu betonen, dass es sich hier primär um Prinzipien der Sinnkonstruktion durch die Rezipienten handelt. Immer wieder einmal kommt der Einwand, dass die Informationen in diesem oder jenem Text, dieser oder jener Textgruppe widersprüchlich oder akausal seien, Erklärungen verweigern, vieldeutig seien usw. Doch damit verfehlt man eine wichtige Pointe des Poetischen: Das Irritationspotential, das solche Texte entfalten können, ist ja gerade ein Indiz dafür, dass die Erwartungen der Rezipienten auf einen Widerstand stoßen. Die Rezipienten sind dann enttäuscht, legen den Text als unverständliches Zeug beiseite - oder begegnen ihm mit besonders hartnäckigen Sinnerwartungen und versuchen, einen entsprechenden Tief-Sinn zu ermitteln. Das sind dann Konstellationen, die ihre Neufunktionalisierung durch den historischen Augenblick erhalten. Die Erzählteleologie z. B. gerät spätestens seit der Darwin-Rezeption in eine tiefe Krise, die anspruchsvolleres teleologisches Erzählen nur noch im Modus der Ironie zulässt (vgl. Ajouri 2007). Und ähnlich wird das Prinzip der Detektion nur noch in der Unterhaltungsliteratur bruchlos realisiert, während anspruchsvollere Autoren das Schema durch Hinterlassen mehr oder weniger provokativer Reste zu verfremden versuchen. Es wird aber natürlich immer noch als Erwartung vorausgesetzt! -

Man kann schon für solche elementaren Erwartungsdispositionen den Begriff der 'Gestalt` verwenden, weil sie jedenfalls das Minimalerfordernis einer apriorischen Selektion nach 'Figur und 'Grund,, Vordergrund und Hintergrund, Relevantem und Nichtrelevantem erfüllen. Dass es sich in diesen Fällen um stammesgeschichtliche Vorgaben handelt, erscheint mir so selbstverständlich, dass ich es hier nicht weiter erörtere. ${ }^{5}$ Im folgenden soll es um etwas weiträumigere Schemata gehen, die mindestens drei Positionen enthalten, also, quasi im aristotelischen Sinn, eine Sequenz von Anfang, Mitte und Ende aus dem Strom der Eindrücke herausheben und überdies auch mit bestimmten inhaltlichen Momenten affin sind. Die Eingangsfrage spezialisiert sich nun: Wie weit geht die stammesgeschichtliche, sozusagen universelle Verwurzelung, welche Varianten eines Grundmusters sind schon von der Disposition her vorgesehen und wo setzen dann die historisch-jeweiligen Variablen ein?

Solche Elementarplots sind immer wieder einmal ermittelt worden, ohne dass dabei eine stammesgeschichtliche Verwurzelung erörtert worden wäre. Thirty-Six

${ }^{5}$ Nicht selbstverständlich sind allerdings einige Konsequenzen, die an anderer Stelle zu behandeln sind. Vgl. jetzt z. B. die Ausführungen zum Zusammenhang von Induktion und Metapher in Eibl 2006 und 2009b. 
Dramatic Situations hat weiland Georges Poltis aufgelistet, ${ }^{6}$ neun Einfache Formen identifizierte Andre Jolles (Jolles 1930), für angehende Autoren hat Ronald B. Tobias 20 Masterplots vorgeschlagen (Tobias 2004), und nun offeriert Christopher Booker Seven basic plots. (Booker 2004). Das sind Unternehmungen von recht unterschiedlicher Struktur und unterschiedlichem Anspruch. Aber gemeinsam ist ihnen der Wunsch nach Elementarisierung, nach einer Zerlegung des komplexen Instrumentariums des Erzählens in nicht weiter reduzierbare Einheiten, die es möglich machen könnte, dem Reden über literarische Sachverhalte etwas von seiner Vieldeutigkeit zu nehmen. Will man sich bei solchen Verfahren der Elementarisierung nicht mit bloßem Aufraffen begnügen, verfährt man tendenziell strukturalistisch oder analytisch, d. h. die Kategorien sind Teil einer Matrix, die nach Kriterien der Vollständigkeit und logischen Verträglichkeit konstruiert wird. Dieses Ideal der Begriffsbildung hat sicher große Verdienste und soll hier gar nicht weiter in Frage gestellt werden. Es weist jedoch ähnliche Grenzen auf wie Linnés System der Pflanzen, das den Gegenstandsbereich nach der Zahl der Staubfäden und der Form der Stempel ordnete, aber die natürlichen Verwandtschaften eher zufällig berücksichtigte. Die folgenden Überlegungen lassen sich am ehesten als biologischer Teil einer korrelativen Typisierung auffassen (ein anderer Teil wäre der soziokulturelle). Die Positionen, die auf diese Weise gewonnen werden, sind weit entfernt von der Vollständigkeit und Geschlossenheit rubrizierender Taxonomien, sind dafür aber selbst Stationen eines erklärenden, nach vorne offenen Forschungsprogramms.

Ich folge zunächst einem Hinweis, den der Altphilologe Walter Burkert gegeben hat. Burkert findet in vielen Werken der Weltliteratur die Formel von der abenteuerlichen Suche`, ergänzt zum Schema von Auszug und Rückkehr, wie Vladimir Propp sie aus russischen Märchen herausdestilliert hat:

Nach Propp lässt sich eine Erzählung als Abfolge von 31 `Funktionen` beschreiben. Verkürzt und vereinfacht verläuft dies so: Es kommt ein Verlust zustande, ein Bedürfnis oder Wunsch (8); der `Held ‘ wird ausgeschickt (9), er fasst seinen Entschluss (10); er verlässt sein Zuhause (11); er begegnet einem Partner, der ihn auf die Probe stellt (12); indem er darauf reagiert (13), erhält er ein Geschenk, ein Zaubermittel (14); so ausgestattet erreicht er den gesuchten Ort (15), wo er mit einem Gegner in Konflikt gerät (16); er erhält dabei eine Markierung, ja Verwundung (17), doch bleibt er siegreich (18); der anfängliche Verlust oder Mangel ist damit behoben (19). Der >Held ‘ tritt die Rückreise an (20); er wird verfolgt (21), doch gerettet (22); er kommt unerkannt nach Hause oder an einen neuen Ort (23); ein falscher Held tritt auf als Konkurrent (24); über eine schwere Probe (25) kommt der `Held zum endlichen Erfolg (26), er wird erkannt (27), der falsche Held wird entlarvt (28), bestraft (30); der `Held vermählt sich und besteigt den Thron (31). (Burkert 1998, 76)

${ }^{6}$ Übersetzt nun bei Tieger 2004. 
Burkert entdeckt dieses Schema - nicht in allen Einzelschritten, aber im groben Ablauf - in der griechischen Mythologie, insbesondere in der Heraklessage, der Argonautensage, Teilen der Odyssee, ebenso im Gilgamesh-Epos und anderen alten Geschichten, geht aber auch bis in die Moderne: „Science Fiction und ComputerSpiele kommen am wenigsten davon los."(ibid., 81). Natürlich ist das Schema und sein häufiges Vorkommen schon öfter beobachtet worden. Bei Tobias oder bei Booker z. B. erscheint es als das Schema der Suche (^Quest $`$ ). Neu an Burkerts Überlegungen ist, dass er die zu vermutende Universalität explizit in einen biologischen Zusammenhang stellt. Er führt Washoe an, eine der ssprechenden Schimpansinnen, mit der sich der folgende Dialog in amerikanischer Gehörlosensprache abspielte: „George: Was willst du? Washoe: Orange, Orange. George: Keine Orange mehr da. Was willst du? Washoe: Orange. George (ärgerlich werdend): Keine Orange mehr da. Was willst du? Washoe: Du Auto gehen. Gib mir Orange. Schnell.« Washoe schickt also ihren Partner aus, damit dieser Futter hole, und entsprechend meint Burkert, die Hauptlinie der Propp-Sequenz sei biologisch durch die praktische Notwendigkeit der Nahrungssuche »vorgezeichnet« (ibid., 84). Vorgezeichnet - es ist nicht zu verkennen, dass Burkerts These ihre Plausibilität nicht zuletzt der Vagheit in diesem Punkt verdankt. Wenn sie als Wegweiser eines Forschungsprogramms dienen soll, wird man sie präzisieren müssen.

Zunächst sind noch andere Angebote anthropologischer Universalisierung zu beleuchten. Dazu gehört z.B. die psychoanalytische Motivfigur. Carl Pietzker hat die Erzählungen von ursprünglicher Einheit, gegenwärtiger Trennung und künftiger wiedergewonnener Einheit in ihren vielfältigen Manifestationsbereichen unter diesem Aspekt untersucht (Pietzcker 1996). Er konnte deutlich machen, wie dieses Grundmuster im religiösen, philosophischen, literarischen und politischen Denken und Leben eine Wirksamkeit entfaltet, die nur mit der Annahme eines sehr elementaren Mechanismus zu erklären ist. Er stößt auch auf ein Grundproblem der Analyse solcher Muster: dass sie nämlich immer aus einer unveränderlichen, suniversalen Komponente und einer kulturell und individuell variablen Komponente bestehen und dass es sehr schwer ist, diese beiden Teile voneinander zu unterscheiden und einander zuzuordnen. Seine Lösung liegt bei der Psychoanalyse, genauer: Bei einer Art universellem Urtrauma, das alle Menschen durchleben, wenn sie den `vortriangulären Raum der Mutter-Kind-Einheit verlassen müssen, und das in Krisensituationen wieder aktiviert und zum Motor von snarzisstischen Paradiesesphantasien wird. Die Krisensituationen in ihrer ganzen denkbaren Vielfalt wären demnach die variable Komponente - vom Verlust eines geliebten Menschen oder eines gewohnten Habitats oder auch nur einem ärgerlichen Regenwetter über drohenden Nahrungsmangel oder drohende Feindesgefahr bis zu den großen historischen und religiösen Orientierungskrisen, wie sie insbesondere im Prozess der Modernisierung der letzten 400 Jahre zu neuen überindividuellen Gemütslagen geführt haben. Und die Paradiesesphantasien, die darauf reagieren, wären entsprechend vielgestaltig, reichten von individuellen Glücksträumen Diesseits- und Jen- 
seits-bezogener Art bis zu den großen religiösen und philosophischen Heilsgeschichten.

Ich erspare es mir und meinen Lesern, hier meine Zweifel an den psychoanalytischen Grundlagen von Pietzckers Argumentation und mein Bedauern über den dogmatischen Glaubensgestus seines im Übrigen sehr klugen Buches auszubreiten. Gerade was die Grundlagen angeht, wäre jedoch darauf hinzuweisen, dass Pietzcker zwei für seine Perspektive sehr einschlägige monumentale Werke ignoriert, nämlich Ernst Blochs an Marx orientiertes Prinzip Hoffnung und die Forschungen des Psychoanalytikers John Bowlby über Bindung und Verlust.

Blochs expressionistische Argumentationsweise mit ihrer Daueraufgeregtheit hat heute stark an Reiz verloren. Gleichwohl ist sein Werk wegen seiner enzyklopädischen Vollständigkeitsintention noch immer eine wertvolle Zusammenstellung des riesigen Feldes von Bereichen des Denkens und Handelns, in denen die Vorstellung einer besseren individuellen und/oder gesellschaftlichen Zukunft ein Rolle spielt. Er mag zwar die Freud'sche Psychoanalyse nicht; hier werde die Libidogeplagtheit der Wiener Müßiggänger von 1900 unzulässig verallgemeinert. Aber bei der Begründung seiner Ablehnung gerät er in ein nicht untypisches Dilemma. Es geht ihm mit Marx um die Umstände, die sich wandeln und die vor allem in verschiedenen sozialen Klassen verschieden sind und deshalb bei der $>$ Leisure Class` und beim Proletariat höchst verschiedene Handlungsantriebe hervorbringen. Die "Angst vor Verlust der Arbeit" sei "schwerlich ein Kastrationskomplex« (Bloch 1967, I 73). Da aber das Prinzip Hoffnung als etwas Überzeitliches konzipiert wird, muss auch Bloch irgendetwas Universelles als Ursache postulieren. Es gebe »keine erotische Geschichtsauffassung an Stelle der ökonomischen, keine Welterklärung aus Libido und ihren Entstellungen statt aus Wirtschaft und ihren Überbauten. Daher bleibe man endlich auch hier beim realen Ausdruck der Sache: Beim wirtschaftlichen Interesse, als dem gleichfalls nicht einzigen, aber grundlegenden. $\ll^{7}$ Der Haupttrieb sei mithin nicht die Libido, sondern der Selbsterhaltungstrieb, nicht die Liebe, sondern der Hunger.

Natürlich ist das keine taugliche Alternative. Denn `Hunger` und `Liebe ' gehören zusammen: Das entscheidende Nadelöhr der biologischen Evolution ist immer die Fortpflanzung, aber um bis zur Fortpflanzung zu kommen, muss man erst einmal überleben. Jedenfalls aber rekurriert Bloch auf eine biologisch zu verstehende Universalie. 'Hunger in diesem Sinne bezieht sich bei ihm aber nicht nur auf Nahrung im literalen Sinn, sondern auf alles, was wir begehren. Immer seien Religionen und die großen Philosophien bestimmt gewesen durch »den Trieb, nach Hause zu gelangen " (ibid. , I 6). Gewiss müssen dann wieder die geschichtlichen Wandlungen des Selbsterhaltungstriebes hervorgehoben werden. Als Machttrieb z. B. erscheine er erst unter den Bedingungen der Klassenteilung. Doch unter der Hand wird aus dem operationalen Begriff der Selbsterhaltung der idealistische Normbegriff der

${ }^{7}$ Ibid. 
Selbstentfaltung: "So bedeutet Selbsterhaltung letzthin den Appetit, unserem sich entfaltenden, erst in und als Solidarität sich entfaltenden Selbst angemessenere und eigentlichere Zustände parat zu halten.« (ibid., I 77). Damit ist das Prinzip Hoffnung als Formel universalisiert und gleichwohl auf jede beliebige historisch-kulturelle Situation beziehbar.

John Bowlby hat versucht, die psychoanalytische Methodik zu modernisieren, und er hat dabei die biologische Unzulänglichkeit der Freudschen Position recht zutreffend charakterisiert. ${ }^{8}$ Er zeigt den historischen Weg, auf dem »Freuds Position innerhalb der theoretischen Biologie völlig in Widerspruch zu der Biologie geraten [war], die das zwanzigste Jahrhundert beherrschen sollte«, und erweitert das Urteil auf die Psychoanalyse insgesamt:

Die Kluft zwischen der übrigen, fest auf einer weiterentwickelten Version der Darwinschen Prinzipien beruhenden Biologie und der lamarckistische Ideen beibehaltenden Psychoanalyse ist seither ständig und unausweichlich tiefer und breiter geworden. Aus diesem Dilemma gibt es nur zwei Auswege. Der erste, der kaum vorstellbar ist, würde bedeuten, dass die Biologie ihre Darwinsche Perspektive aufgibt. Der zweite, der hier vertreten wird, hieße, dass die Psychoanalyse im Sinne einer modernen Evolutionstheorie umgeformt wird. Die andere Möglichkeit wäre, dass die derzeitige Scheidung der beiden auf unbestimmte Zeit bestehen bleibt, was für die Psychoanalyse hieße, dass sie dauernd hinter den äußersten noch vertretbaren Grenzen der wissenschaftlichen Welt zurückbleibt. (Bowlby 2006, II 332)

In John Bowlbys eigener Bindungstheorie ist die erste menschliche Beziehung des Kindes, und das ist in der Regel die Beziehung zur Mutter, von maßgeblicher Bedeutung für alle späteren Bindungen. Insoweit stimmt seine Position mit anderen Strömungen der Psychoanalyse überein. Er legt jedoch großen Wert auf die biologische Funktion des Bindungsverhaltens. Er wendet sich gegen die Freud'sche Deutung der Mutterbindung als `Sekundärtrieb ${ }^{9}$ und gegen die Neigung vieler Psychoanalytiker, bei jeder Art von Bindung Regression und narzißtische Kränkungen zu entdecken und sie auf diese Weise mehr oder minder deutlich zu pathologisieren. "Wenn man Bindungsverhalten bei einem Erwachsenen als regressiv bezeichnet, vergisst man die lebenswichtige Rolle, die dasselbe von der Wiege bis zum Grabe im Menschenleben spielt." (Bowlby 2006, I 204).

Bowlbys Perspektive ist allerdings etwas einseitig. Er behandelt zwar Bindung, aber Trennung kommt bei ihm nur als Verlust vor, also als etwas, das einem zustößt und unter dem man leidet. Für die Perspektive des Therapeuten ist das gewiss sinnvoll, aber für unsere Zwecke ist eine wichtige Ergänzung zu machen: Neben dem Bindungstrieb oder -instinkt gibt es auch einen genuinen Trennungstrieb, ein Fundamentalbedürfnis nach Unabhängigkeit und Autonomie. Auch hier handelt es sich, wie Norbert Bischof in seinem `Zürcher Modell sozialer Motivation` gezeigt

${ }^{8}$ Zusammengefasst im Anhang von Bindung und Verlust II unter dem Titel »Psychoanalyse und Evolutionstheorie«, $330 \mathrm{ff}$.

9 Vgl. hierzu besonders den Anhang »Über das Wesen der Mutter-Kind-Bindung: Überblick über die psychoanalytische Literatur", I 343-356. 
hat, um einen funktional wertvollen und damit evolutionär erklärbaren Verhaltensantrieb (Bischof 1997). Nicht nur werden auf diese Weise neue Habitate und Ressourcen erschlossen, sondern Trennung ist auch eine wichtige Methode der Konfliktvermeidung und spielt insofern gleichfalls eine lebenswichtige Rolle. Wenn z. B. der Nachwuchs geschlechtsreif wird, ist es ein in der Natur weitverbreitetes Mittel der Inzestvermeidung und der genetischen Durchmischung, dass er das Rudel verlässt und sein `Glück` in der Ferne sucht. Das gilt für die männlichen Nachkommen, die in polygynen Rudeln überdies oft vom Herrscher als potentielle Rivalen zum Weggehen sermuntert werden, aber auch für weibliche Rudelmitglieder, die, mit Schnitzler zu reden, dem Ruf der Hirtenflöte folgen und sich schließlich einem anderen Rudel anschließen. Bindungstrieb und Trennungstrieb (Sicherheitsbedürfnis und Erregungsappetenz) sind schon im vormenschlichen Bereich, ja letztlich mit der geschlechtlichen Vermehrung und dem Prinzip der Heterozygotie gesetzt. ${ }^{10}$

3.

Umso dringender stellt sich damit aber die Frage, weshalb das zur Grundlage von Geschichten wird. Man muss sich für die Relevanz dieser Frage erst einmal die Unwahrscheinlichkeit des Sachverhalts verdeutlichen. Dass es einen Bindungstrieb und einen Trennungstrieb gibt, bedeutet ja keineswegs, dass wir Geschichten erzählen müssen, die von diesen Trieben handeln. Sie wirken eben, das könnte ja genügen, und bei unseren nichtmenschlichen Verwandten genügt es ja tatsächlich. Aber die Menschen reden unablässig, müssen schlechterdings über alles reden, was ihnen begegnet. Natürlich liegt das an der Fähigkeit, zu sprechen, aber evolutionär gesehen verdanken sich Fähigkeiten immer einem Reproduktionsvorteil, der als Selektionsfaktor wirkte, also einem Selektionsdruck. Welchen Reproduktionsvorteil bringt es, wenn man Geschichten über Ausfahrt und Heimkehr oder Bindung, Trennung und Wiederfinden erzählt?

Die Antwort kann in zwei Stufen erfolgen. Generell gilt, dass durch wiederholte und variierte typische Situationen die Triebe modelliert werden. Diese Situationen mögen aus eigener Erfahrung wahrgenommen werden oder durch Mitteilung der Erfahrungen anderer oder durch erfundene Geschichten. Erzählungen über Bindung und Trennung, aber auch über Sexualität, Aggression usw. ermöglichen es, die Triebe zu entspezialisieren und als Problemlösungen für eine Vielzahl von heterogenen, aber analogen Problemen zu programmieren. Das ist ein ganz wesentliches Moment dessen, was wir unter menschlicher Kultur verstehen. Die umstrit-

${ }^{10}$ Um die Vielfalt der Triaden zu signalisieren, weise ich hier auf die Grundstruktur der Übergangsriten im Sinne von van Gennep 1986 hin. Diese Übergangsriten kennen eine Ablösungsphase (mit »rites de séparation"), eine Zwischen- und Umwandlungsphase (mit "rites de marges») und eine Integrationsphase (mit »rites d'aggregation«). 
tene evolutionspsychologische Lehre von der Modularität der Adaptationen ist ja eigentlich eine Lehre von der modularen Entstehung der Adaptationen. Ein Beispiel ist der >Cheater Detector . Wenn es über viele Generationen hin einen Reproduktionsvorteil bietet, dass man Betrüger entlarvt, dann entsteht ein 'Cheater Detector-Modul, also ein kognitives Instrument, mit dem Betrüger entlarvt werden können. ${ }^{11}$ Cosmides/Tooby konnten empirisch nachweisen, dass logische Schlussverfahren dann besonders gut funktionieren, wenn es darum geht, einen Täuschungsversuch aufzudecken. Es wird die empirisch arbeitenden Psychologen nicht viel Raffinement kosten, Vergleichbares auch für Nahrungssuche und -auswahl, Jagdtechniken, Werkzeugherstellung, Wetterprognosen usw. zu belegen, also für weitere lebenswichtige Betätigungen der Sammler und Jäger und ihrer Vorfahren. Unsere Fähigkeit zu logischem Denken hat sich aufgrund des Selektionswertes solcher stereotyper praktischer Aufgaben entwickelt. Da sich dieses Instrument aber in sehr vielen Lebenssituationen bewähren konnte, hat es sich zum Mehrzweck-Instrument entwickelt, das auch in analogen, doch evolutionär neuen Problemlagen eingesetzt werden konnte. So ähnlich kann man sich die Erweiterung der modularen sdedicated intelligencer zur allgemeineren simprovisational intelligencer vorstellen. ${ }^{12}$

Ein entscheidender Entwicklungsantrieb dürfte von der Möglichkeit der sprachlichen Vordefinition ( - Probehandeln) von relevanten Situationen ausgegangen sein. Hier liegt dann der evolutionäre Vorteil des Erzählens: Es kann vielseitige, sunpräzise Adaptationen (`offene Instinktprogramme im Sinne Mayrs [Mayr 1991]) mit einer Skala von Festlegungsmöglichkeiten ausstatten und diese dann durch entsprechendes Wiederholen ins Gedächtnis einprägen. Da kann dann die ganze Skala der Bindungsmöglichkeiten von der Mutter über die Horde bis zur Nation ebenso eingesetzt werden wie die ganze Skala der Trennungsmöglichkeiten, vom Entwöhnen des Säuglings über das Verlassen des Elternrudels oder des Elternhauses bis zur Rebellion der Jungen oder der Suche nach dem Heiligen Gral.

Damit ist aber nur die hohe Variabilität der beiden Grundpositionen des Schemas erklärt, nicht jedoch die Verknüpfung dieser Positionen zu einem dreistelligen Standard-Plot. Worin liegt dessen evolutionärer Vorteil? Er kann nur aufgefunden werden als Unterstützung einer vorteilhaften Handlungsdisposition. Man kann hier durchaus Blochs Formel vom >Prinzip Hoffnung heranziehen. Es dürfte unmittelbar einleuchtend sein, dass dieses Prinzip - zumindest statistisch gesehen - in Situationen der Bedrängnis eher zu einer Problemlösung beiträgt als ein Prinzip Hoffnungslosigkeit. (Dieses kann nur dann hilfreich sein, wenn durch seine Äußerung die Unterstützung von Schutzpersonen provoziert wird, d. h. als supplikatives Problemlösung - aber dann ist es wieder eine verkappte Variante des Prinzips Hoff-

\footnotetext{
${ }^{11}$ Der Sachverhalt ist mehrfach beschrieben worden, u. a. in Cosmides/Tooby 1992.

12 Dazu grundlegend Cosmides/Tooby 2001.
} 
nung.) Voraussetzung für das Wirken eines solchen Prinzips und die Begründung einer Erzählsequenz ist aber, dass zwischen Problemwahrnehmung und Problemlösung eine Phase der Reflexion und Lagebeurteilung liegt. Die bloße ,fest verdrahtete Reaktion auf einen Reiz kommt ohne Hoffnung aus. Man wird, mit aller Vorsicht, sagen dürfen, dass diese Phase der Reflexion beim Menschen jedenfalls ausgedehnter ist als bei allen anderen Lebewesen. ${ }^{13}$ Nur so lassen sich Geschichten erzählen. Oder anders gesagt: Nur so lassen sich Ereignisse in eine 'Gestalt ‘ bringen, die über den augenblicklichen Zustand und die augenblickliche Reiz-ReaktionsKonstellation hinaus so etwas wie Anfang und Ende mitentwerfen, also die Gegenwart zeitlich einbetten.

Das Repetieren angeborener Plots hat anscheinend die Funktion, ein dahinterliegendes Schema als kognitive Weltstrukturierung zu bestätigen und variierend einzuüben. Die kognitive Weltstrukturierung ließe sich etwa umschreiben als: Es wird wieder so werden, wie es einmal war. Und die variierende Einübung bestünde darin, dass das Schema in einer möglichst großen Breite möglicher Anwendungsbereiche paratgehalten wird. Von besonderer Bedeutung sind dabei natürlich Geschichten von Ausfahrt und Heimkehr. Auf ähnlich elementare Nöte und Bedürfnisse beziehen sich Geschichten von Krankheit und Heilung. Die politische Geschichte ist voll von Wiedergewinnungen einer angeblichen alten Heimat, von Wiedervereinigungen getrennter Völker oder Sippen. Die Wiederentdeckung oder -erkennung verschollener Personen gehört ebenso hierher wie das Wiederfinden verlorener oder verschwundener Gegenstände. Selbst dass der verlassene Liebhaber sich meistens eine Zeitlang die Wiedergewinnung der Geliebten erträumt, sie womöglich mit Gewalt herbeizuführen versucht, hat seine Ursache in einer evolutionären Konstellation: Man möchte zwar meinen, es sei generativ zweckmäßiger, wenn er sich möglichst schnell eine neue Partnerin sucht, aber zunächst ist es erfolgverheißender, wenn er die alte Beziehung noch einmal zu retten versucht. Und schließlich wollen wir nicht die Welt der Religionen und mythischen Erzählungen vergessen, die das Prinzip von Trennung und Wiedervereinigung gar als Weltprinzip verankern.

Wenn wir also aus dem Dargelegten folgern wollen, was nun eigentlich der evolutionär entstandene, angeborene Kern der triadischen Erzählungen ist, dann ist es das Schema von vergangener Problemlosigkeit, gegenwärtigem Problemdruck und künftiger Wiederkehr der Problemlosigkeit. Dieser Kern ist bereits das Ergebnis eines Prozesses, den man als eine phylogenetische Induktion (oder Abstraktion) bezeichnen könnte: Eine Vielzahl heterogener, doch analoger Probleme und Problemlösungsstrategien kann zu einem übergeordneten Lösungsalgorithmus zusammengefasst werden. Auf diese Weise entsteht die Möglichkeit simprovisierender Intelligenzı, die sogar völlig neue, doch in relevanten Grundzügen analoge Probleme lösen kann. Kehrseite dieser Entroutinisierung ist die Gefahr völliger Orientie-

${ }^{13}$ Hierzu schon die epochemachenden Untersuchungen von Köhler 1963. 
rungslosigkeit, eine Folgerungslücke zwischen dem Allgemeinen des Problemlösungsalgorithmus und dem Besonderen der Anwendungssituation. Um diese Lücke zu schließen, entwickeln die Kulturen eine ganze Skala von Techniken zur Einübung möglicher Applikationstypen - darunter die Geschichten von Ausfahrt und Heimkehr, Trennung und Wiedersehen, Verlieren und Finden, Erkranken und Genesen, die das Grundschema lebendig erhalten und jene Hoffnung und Zuversicht einüben, die zur Lösung der jeweils anstehenden Probleme antreiben können.

Karl Eibl Institut fir deutsche Philologie Ludwig-Maximilians-Universität München

\section{Literatur}

Philip Ajouri, Erzählen nach Darwin. Die Krise der Teleologie im literarischen Realismus: Friedrich Theodor Vischer und Gottfried Keller, Berlin 2007.

Norbert Bischof, Das Rätsel Ödipus. Die biologischen Wurzeln des Urkonflikts von Intimität und Autonomie [1985], München ${ }^{5} 1997$.

Ernst Bloch, Das Prinzip Hoffnung, 3 Bde., Frankfurt a. M. 1967.

John Bowlby, Bindung und Verlust, 3 Bde., München 2006.

Christopher Booker, Seven Basic Plots. Why we tell stories, London/New York 2004.

Walter Burkert, Kulte des Altertums. Biologische Grundlagen der Religion, München 1998.

David M. Buss, Wo warst du? Vom richtigen und falschen Umgang mit der Eifersucht, Kreuzlingen/München 2000.

Leda Cosmides/John Tooby, Cognitive Adaptations for Social Exchange, in: Jerome H. Barkow/Leda Cosmides/John Tooby (Hg.): The Adapted Mind. Evolutionary Psychology and the Generation of Culture, New York 1992, 163-228.

-, Unraveling the enigma of human intelligence. Evolutionary psychology and the multimodular mind, in: R. J. Sternberg/J. C. Kaufman (Hg.), The evolution of intelligence, Hillsdale 2001.

E. Bruce Goldstein, Wahrnehmungspsychologie. Zweite deutsche Auflage herausgegeben von Manfred Ritter, Heidelberg/Berlin 2002.

Irenäus Eibl-Eibesfeldt/Christa Sütterlin, Weltsprache Kunst. Zur Natur und Kunstgeschichte bildlicher Kommunikation, Wien 2007.

Karl Eibl, Eine Kuh ist eine Ziege. Zu den evolutionsbiologischen Wurzeln der Metaphorik, Der Deutschunterricht 6:06 (2006), 44-52.

—, Kultur als Zwischenwelt. Eine evolutionsbiologische Perspektive, Frankfurt a. M. 2009 (Eibl 2009a).

-, The Induction Instinct. Evolution and Poetic Application of a Cognitive Tool, Studies in the Literary Imagination 42:2 [erscheint 2009] (Eibl 2009b).

Andre Jolles, Einfache Formen. Legende, Sage, Mythe, Rätsel, Spruch, Kasus, Memorabile, Märchen, Witz [1930], Darmstadt ${ }^{2} 1958$.

Wolfgang Köhler, Intelligenzprüfungen an Menschenaffen [1921], Berlin/Göttingen/Heidelberg $^{2} 1963$. 
Ernst Mayr, Eine neue Philosophie der Biologie, München/Zürich 1991.

Carl Pietzcker, Einheit, Trennung und Wiedervereinigung. Psychoanalytische Untersuchungen eines religiösen, philosophischen und literarischen Musters, Würzburg 1996.

Karl R. Popper, Naturgesetze und theoretische Systeme, in: Hans Albert (Hg.), Theorie und Realität [1964], Tübingen ${ }^{2} 1972$.

Martin Schuster, Kunstpsychologie. Kreativität, Bildkommunikation, Schönheit, Hohengehren 2000.

Gerhild Tieger, Lass Laufen! Beats, Wendepunkte, Krisen \& Konflikte. Mit den 36 dramatischen Situationen von Georges Polti, Berlin 2004.

Ronald B. Tobias, 20 Masterplots. Woraus Geschichten gemacht sind, Frankfurt a. M. 1999.

Arnold van Gennep, Übergangsriten [frz. Original 1909], Frankfurt a. M. 1986.

Oskar Walzel, Gehalt und Gestalt im Kunstwerk des Dichters [1929], Nachdruck Darmstadt 1957. 